Methods The 'Research for Health Justice Framework' makes recommendations on how global health research partnerships may foster the ideals of justice through their selection of research populations and questions, research capacity strengthening, delivery of ancillary care and the provision of post-trial benefits. We applied these criteria to collaborative genomics research consortia in Africa (an example of global health research in Africa).

Results The results show that the lack of national health research priorities in most African countries hinders the intention of global health actors to use global health research as a means of promoting global health equity. Furthermore, capacity building efforts need to be more coordinated and monitored. The responsibility for this lies with several actors. Conclusion The potential for global health research to improve the health capability of countries in Africa will require that attention is paid to research that improves the health of people in Africa and that global health research partnerships identify, first and foremost, what kind of capacity strengthening is required and who is responsible for this activity. African governments and research institutions can play a role to help global health research improve health and health equity in Africa, in ways that are sustainable.

\section{OC 8378 REQUIREMENTS FOR THE ETHICAL CONDUCT OF CLINICAL RESEARCH IN CAMEROON, SUB-SAHARAN AFRICA: THE IMPORTANCE OF ACTIVE MONITORING}

${ }^{1}$ Marceline Djuidje Ngounoue*, ${ }^{2}$ Jerome Ateudjieu, ${ }^{1}$ Charles Fokunang, ${ }^{3}$ Primus Che Chi, ${ }^{1}$ Mireille Ndje Ndje, ${ }^{1}$ Sylvie Kwedi Nolna, ${ }^{4}$ Gisele Magne, ${ }^{5,6}$ Lazare Kaptue. ${ }^{1}$ University of Yaounde I, Cameroon; ${ }^{2}$ University of Dschang, Cameroon; ${ }^{3}$ Peace Research Institute Oslo (PRIO), Norway; ${ }^{4}$ Clinique Bastos, Cameroon; ${ }^{5}$ Universite des Montagnes, Bangangte, Cameroon; ${ }^{6}$ Cameroon National Ethics Committee, Cameroon

\subsection{6/bmjgh-2019-EDC.8}

Background Previous evaluation of the state of ethics regulation in Cameroon revealed: law regulating clinical research is lacking; existing committees lack infrastructure and financial support to sustainably review and effectively monitor approved protocols. The present Cameroon National Ethics Committee (CNEC)-EDCTP project aimed at implementing and evaluating active monitoring of clinical research in Cameroon.

Methods Between 2011-2013, approved clinical trials and protocols involving transfer of biological materials abroad were consecutively monitored. The monitoring tool, a questionnaire on the conformity of key documents e.g. research protocols, ethical clearance, informed consent documents, investigator's brochure, with a focus on GCP standards was sent to promoters/investigators ten days prior to the field visit. Teams of two-three monitors, made up of CNEC members and independent consultants, were mobilised per site (hospital/research institute/NGO). Reports with key recommendations were submitted to CNEC for review and approval, to different promoters/investigators, and the Regulatory Authority for action; the monitoring summary was submitted to EDCTP.

Results Up to 22 site visits were done throughout the country, monitoring about 30 protocols within 11 hospitals, 9 research institutes, a National Programme and a non-governmental organisation. All sites had ethical clearance and administrative authorisation for research. From the registered number of research participants, less than half of the signed consent forms were available. Other issues were the lack of full involvement of local investigators, inexistence of material transfer and data sharing agreements with collaborative studies, non-implication of study communities/participants. As educators-consultants, monitors formulated recommendations to investigators, ethics committees and regulatory authority, insisting on the implication of local PI/collaborators with defined percentages of time to be devoted for research and good participatory practice among research communities/participants. Conclusion Active monitoring shows some formality in the application of ethical/administrative clearance in Cameroon. However, complex issues raised confirm the necessity of continuous monitoring to meet the high standards for clinical research ethics in Cameroon.

\section{OC 8391 AFREENET (AFRICA ETHICS EXCELLENCE NETWORK): A NETWORK OF NATIONAL RESEARCH ETHICS COMMITTEES ENGAGED IN THE REINFORCEMENT OF THEIR CAPACITIES}

${ }^{1}$ Virginie Pirard, ${ }^{2}$ Louis Penali, ${ }^{4}$ Armande Gangbo, ${ }^{3}$ Oumou Younoussa Sow, ${ }^{1}$ Samira Ouchhi*. ${ }^{1}$ Institut Pasteur, Paris, France; ${ }^{2}$ National Ethics Committee for Health Research, Côte d'Ivoire; ${ }^{3}$ National Ethics Committee for Health Research, Conakry, Guinea; ${ }^{4}$ National Ethics Committee for Health Research, Benin

10.1136/bmjgh-2019-EDC.9

Background The establishment of research ethics committees (RECs) in charge of reviewing research protocols answers the need to regulate with an ethical framework the development of clinical trials, biomedical research and technologies affecting human health. The first RECs were instituted at the national level in the $60 \mathrm{~s}$, and were gradually put in place in Africa as a result of research projects development in the area of epidemics such as HIV infection, and to meet one of the major requirements of the Helsinki Declaration and the international ethical Guidelines CIOMS: 'each research protocol involving humans has to be reviewed by an independent ethics committee'. However, RECs in Africa are still facing various challenges in the accomplishment of their missions. Among them, RECs located in the West-African French speaking area are reporting an urgent need for networking and coordinating their effort.

Supported by EDCTP, AFREENET (AFRica Ethics Excellence NETwork) is a 3 year collaborative project between three RECs, respectively in Côte d'Ivoire, Guinea and Benin, and coordinated by the Ethics Unit of Institut Pasteur (France), which aims to reinforce RECs capacities.

The project will lead to reinforcement of training capacities through a long-term strategy to establish mechanisms to ensure and update members' training, and to develop a pool of ethics trainers. Based on RECs experiences, network activities will permit to share and identify valuable practices on global ethics oversight, such as monitoring, SOPs, regulatory issues and sensitisation. The preparation of Ethics Committees for the potential occurrence of outbreaks will be specifically addressed through an Outbreak preparation plan built upon lessons learnt from the Ebola crisis.

Creating synergies and mutual empowerment between African RECs will increase their visibility, their capacity for advocacy and their recognition as key actors of a responsible ethics research framework at the national, regional and international level. 